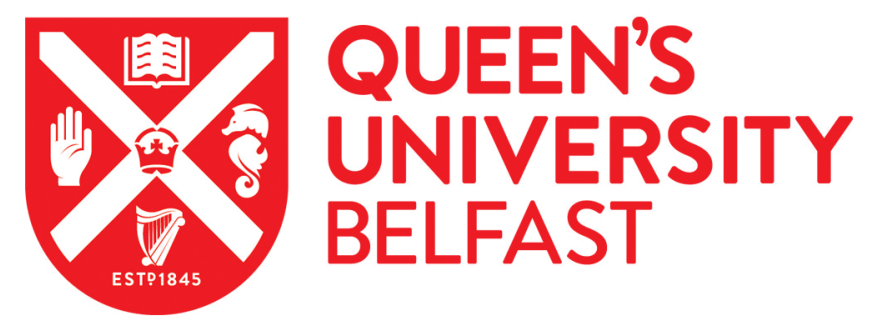

\title{
Synchrophasor-Based Islanding Detection for Distributed Generation Systems Using Systematic Principal Component Analysis Approaches
}

Guo, Y., Li, K., Laverty, D. M., \& Xue, Y. (2015). Synchrophasor-Based Islanding Detection for Distributed Generation Systems Using Systematic Principal Component Analysis Approaches. leee Transactions On Power Delivery, 30(6), 2544 - 2552. https://doi.org/10.1109/TPWRD.2015.2435158

Published in:

leee Transactions On Power Delivery

Document Version:

Peer reviewed version

Queen's University Belfast - Research Portal:

Link to publication record in Queen's University Belfast Research Portal

Publisher rights

Copyright 2015 IEEE. Personal use of this material is permitted. Permission from IEEE must be obtained for all other users, including reprinting/ republishing this material for advertising or promotional purposes, creating new collective works for resale or redistribution to servers or lists, or reuse of any copyrighted components of this work in other works.

\section{General rights}

Copyright for the publications made accessible via the Queen's University Belfast Research Portal is retained by the author(s) and / or other copyright owners and it is a condition of accessing these publications that users recognise and abide by the legal requirements associated with these rights.

Take down policy

The Research Portal is Queen's institutional repository that provides access to Queen's research output. Every effort has been made to ensure that content in the Research Portal does not infringe any person's rights, or applicable UK laws. If you discover content in the Research Portal that you believe breaches copyright or violates any law, please contact openaccess@qub.ac.uk. 


\title{
Synchrophasor-Based Islanding Detection for Distributed Generation Systems Using Systematic Principal Component Analysis Approaches
}

\author{
Y. Guo, Student Member, IEEE, K. Li, Senior Member, IEEE, D.M. Laverty, Member, IEEE, and Y. \\ Xue, Member, IEEE
}

\begin{abstract}
Systematic principal component analysis (PCA) methods are presented in this paper for reliable islanding detection for power systems with significant penetration of distributed generations (DGs), where synchrophasors recorded by Phasor Measurement Units (PMUs) are used for system monitoring. Existing islanding detection methods such as Rate-of-change-offrequency (ROCOF) and Vector Shift are fast for processing local information, however with the growth in installed capacity of DGs, they suffer from several drawbacks. Incumbent genset islanding detection cannot distinguish a system wide disturbance from an islanding event, leading to maloperation. The problem is even more significant when the grid does not have sufficient inertia to limit frequency divergences in the system fault/stress due to the high penetration of DGs. To tackle such problems, this paper introduces PCA methods for islanding detection. Simple control chart is established for intuitive visualization of the transients. A Recursive PCA (RPCA) scheme is proposed as a reliable extension of the PCA method to reduce the false alarms for time-varying process. To further reduce the computational burden, the approximate linear dependence condition (ALDC) errors are calculated to update the associated PCA model. The proposed PCA and RPCA methods are verified by detecting abnormal transients occurring in the UK utility network.
\end{abstract}

Index Terms-ROCOF, Principal Component Analysis, Recursive PCA, Islanding detection, Phasor Measurement Unit, Synchrophasors, Distributed Generation.

\section{INTRODUCTION}

$\mathbf{R}$ ENEWABLE energy sources such as wind and solar power, have become the fastest growing sources of electricity in many countries and regions. Some networks experienced instants of significant level of renewable infeed, i.e. $50 \%$ in Ireland to the gross electricity consumption from time to time. [1], [2]. Considerable impact has been brought on the power system planning, stability, operation and protection [3], of which the unintentional islanding is a key issue to be addressed and it can be dangerous to utility workers and equipments.

Conventional generator islanding detection techniques such as the rate-of-change-of-frequency (ROCOF) [4] method were

This work was financially supported in part by UK EPSRC under grant EP/L001063/1 and China NSFC under grants 51361130153, and in part by the Chinese Scholarship Council.

Y. Guo, K. Li, and D.M. Laverty are with the Department of Electronics, Electrical Engineering and Computer Science, Queen's University Belfast, Belfast, BT9 5AH, UK. (e-mail: yguo01@qub.ac.uk; k.li@qub.ac.uk; david.laverty@qub.ac.uk).

Y. Xue is with the State Grid EPRI, Nanjing, China. (email: xueysh@cae.cn). Corresponding author: Kang Li, (email: k.li@qub.ac.uk). developed when the installed capacity of Distribution Generations (DG) had a negligible impact on system operation, thus known deficiencies in the techniques could be discounted. The threshold for triggering a circuit breaker usually depends on the strength of an actual power system. Along with the increasing penetration of DGs, distribution network begin to present some features which are similar to the transmission network, such as bi-direction power flow within the distribution and upstream network, and back-synchronization after islanding, etc. Therefore, islanding detection for such a distribution network is no longer just a local issue, and the conventional methods are providing unreliable results for power systems with a number of system non-synchronous penetrations (SNSP) as the system does not have sufficient inertia to limit frequency divergences in the event of system fault/stress. This is particularly the case for power grids with limited geographic scope, such as island of Ireland and island of Great Britain, the distance between customers and main grid is not so far away, thus the disturbance caused by main grid more likely affect the distribution network. Large frequency transients caused by loss of bulk generation are causing ROCOF relays to trip as though they are experiencing a local islanding event, even though the fault is far away across the system [5].

The last decade has seen a surge of the deployment of phasor measurement units (PMUs) for wide-area monitoring. PMU technology was historically limited to transmission system applications due to the cost of early devices. However, recent development across the electronics sector has dramatically reduced the price of PMU components. Consequently, PMUs have become an attractive tool across the utility, including distribution systems and embedded generations [6]. [7] proposed a PMU based method to use frequency and angle difference to analyse islanding cases occurred in the North American power grid. The detection ability highly depends on the empirical thresholds of time and frequency change. Other hybrid or novel islanding detection methods using intelligent techniques have also been proposed recently [8], [9]. In our previous work [1], a prototype detector has been demonstrated to outperform the incumbent anti-islanding detectors. Further, in [5], the proposed PMU device has been proved to address the limitations of the incumbent methods, providing a solution that is free of nuisance tripping. However, the threshold settings were also recommended empirically based on data acquired from the Great Britain and Ireland power systems. Further, 
the choice of a reference signal also has a significant impact on the validity of the threshold.

Inspired by the statistical way of analysing industrial process data, principal component analysis (PCA) based fault detection methods have been introduced into islanding detection in power systems [10]-[12]. PCA is able to provide an effective means to choose multiple reference sites across the network, which can process the data collected from PMUs at different locations simultaneously, thus the situation can be avoided that some reference sites are themselves nonsynchronous. Further, for the PCA method, the detection thresholds can be automatically calculated based on multiple references, improving the detection reliability and accuracy. Our previous work [10] has proved the capability of the PCA method for automatic anti-islanding detection when it is applied to the real system PMU data. [11] further demonstrated the advantages of the PCA methods with the focus on the geometric interpretation. [13] extended the work using probabilistic PCA in consideration of the missing values in the PMU measurements. However, the monitoring results using standard PCA method had a number of false alarms due to the slow but normal changes that real system is undergoing. In order to develop more accurate and suitable on-line monitoring scheme for islanding detection, this paper will develop a recursive principal component analysis (RPCA) method to update the training data and the monitoring threshold adaptively. The proposed method can be potentially applied in the future power network with higher level of penetrations of DGs, thus different micro-grids and DGs can be well coordinated especially when faults occur in the main grid. Moreover, grid re-connection after the fault inception can be well organized to prevent further damage. In summary, this paper has mainly three contributions: 1) the threshold settings can be calculated and updated adaptively using recursive method; 2) false alarm rate can be further reduced; and 3) a common wide-area view of the power network can be provided to all dispatchers. Therefore, the proposed methods are of significant theoretic potentials and practical values to improve the reliability and economic efficiency of distribution networks and power systems with high penetration of renewable energies and to protect the customer connected equipment and utility personnel.

This paper is organized as follows. Section II gives an introduction to the synchrophasor based islanding detection scheme. Section III briefly reviews the PCA method. Section IV details the proposed RPCA technique and the systematic monitoring scheme. Section V gives the event analysis followed by experimental results using the proposed PCA methods, in comparison with the conventional ROCOF method. Results of ALDC and RPCA monitoring scheme are also given. Finally, Section VI concludes the paper.

\section{Synchrophasor BASEd ISLANDING DETECTION}

In the UK, islanding detection is governed by Engineering Recommendation G59/3 [14]. This standard is concerned with specifications for conventional methods of islanding protection, but does make allowance for new forms of protection. To facilitate research work on islanding detection, Queen's
University Belfast (QUB) operates an extensive PMU network across both the island of Ireland and the island of Great Britain, as shown in Fig.1.

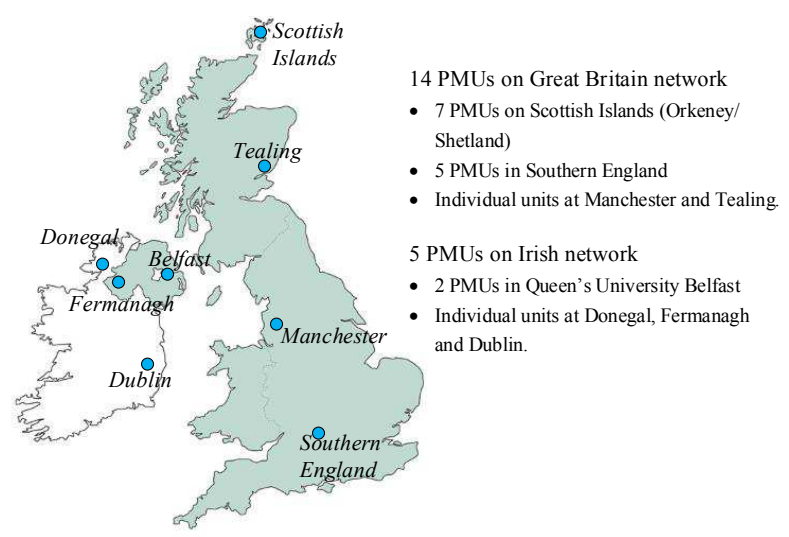

Fig. 1. Synchrophasor islanding detection scheme and PMU locations [1]

PMUs of the OpenPMU design [6] are installed at low voltage $(415 \mathrm{~V})$ at distributed generator locations, and at medium voltage $(33 \mathrm{kV})$, to provide reference synchrophasors from the bulk system. Synchrophasors, containing estimations of voltage amplitude, phase and frequency, are communicated using secure Internet Protocol technologies to a server system located at QUB. This data is analysed in this paper.

\section{INTRODUCTION TO PCA}

\section{A. Conventional PCA}

The raw data consisting of $m$ variables and $n$ samples, is stacked into a matrix $\mathbf{X} \in \mathbb{R}^{n \times m}$. PCA decomposes the normalized data matrix into a score matrix $\mathbf{T} \in \mathbb{R}^{n \times k}$ and a loading matrix $\mathbf{P} \in \mathbb{R}^{m \times k}$ ( $k$ is the number of retained principal components (PCs), $k \leq m$ ) [15]. PCA can decompose the observation matrix $\mathbf{X}$ into the following form:

$$
\mathbf{X}=\mathbf{t}_{1} \mathbf{p}_{1}^{T}+\mathbf{t}_{2} \mathbf{p}_{2}^{T}+\ldots+\mathbf{t}_{k} \mathbf{p}_{k}^{T}+\mathbf{E}=\mathbf{T P}^{T}+\mathbf{E}
$$

where $\mathbf{t}_{i}$ are score vectors, $\mathbf{p}_{i}$ are the loading vectors and $\mathbf{E}$ is the residual matrix. The important statistic for PCA monitoring is given by the Hotelling's $T^{2}$, which is the sum of normalized squared scores defined as [16]:

$$
T_{i}^{2}=\mathbf{t}_{i} \boldsymbol{\lambda}^{-1} \mathbf{t}_{i}^{T}=\mathbf{x}_{i} \mathbf{p} \boldsymbol{\lambda}^{-1} \mathbf{p}^{T} \mathbf{x}_{i}^{T}
$$

where $\mathbf{t}_{i}$ is the $i$ th row of $k$ score vectors from PCA model, and $\lambda^{-1}$ is a diagonal matrix containing the $k$ eigenvalues. The other statistic Q or the squared prediction errors (SPE) is defined by [17]:

$$
Q_{i}=\sum_{j=1}^{m}\left(x_{i j}-\hat{x}_{i j}\right)^{2}
$$

where $x_{i j}$ is the $i$ th measurement value of the $j$ th variable, while $\hat{x}_{i j}$ is the estimated value. When the process is normal, this value should be small. Equations for confidence limit of $T_{\alpha}^{2}$ and $Q_{\alpha}$ can be found in [18] and [19]. A geographical interpretation can better illustrate how PCA works for fault detection, as shown in Fig. 2 [11]. The '•' data represents 
in-control normal operations with two statistics stay in the confidence range. If some sudden abnormal deviations occur on system variables, which are caused by, for example, a mismatch between load and generation, the sum of squares of the distances from the origin along the principal component line to the projected data points will be larger than the upper limit. The aforementioned $T^{2}$ statistic is able to detect such violations, which are represented by ' $\circ$ ' in Fig. 2. On the other hand, a change to the correlation among the variables will increase the projection on the residual subspace. As a result, the total variation in the residual space will violate the confidence limit, which may be caused by the deviation of the mean value from the target, or one of the process variables behaves differently from others. To summarize, for islanding detection, regions in Fig. 2 with data points of ' $\triangle$ ' and ' + ' indicate islanding events.

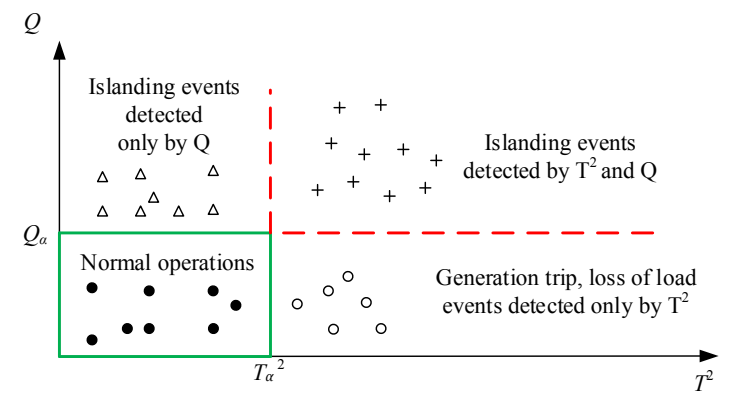

Fig. 2. A geometric interpretation for islanding detection using PCA method, revised from [11].

\section{B. Fault Reconstruction}

In the presence of a fault $\mathbf{F}_{i}$, the estimation $\hat{\mathbf{x}}_{i}$ of the sample vector $\mathbf{x}_{i}$ can be represented using a fault direction vector $\xi_{i}$ as $\hat{\mathbf{x}}_{i}=\mathbf{x}_{i}-\hat{f}_{i} \xi_{i}$, where $\hat{f}_{i}$ is an estimate of the fault magnitude $f_{i}$ which measures the displacement in the direction $\xi_{i}$. Let $\xi_{i}^{T}=[1,0, \ldots, 0]$ so that it can represent a failure of the first process variable in the sample vector $\mathbf{x}_{i}$. The distance between $\hat{\mathbf{x}}_{i}$ and principal subspace is given by the magnitude of the residual vector $\left\|\tilde{\mathbf{x}}_{i}\right\|^{2}$, or the SPE of $\hat{\mathbf{x}}_{i}$.

$$
S P E_{i}=\left\|\tilde{\mathbf{x}}_{i}\right\|^{2}=\left\|\tilde{\mathbf{x}}-f_{i} \tilde{\xi}_{i}\right\|^{2}=\left\|\tilde{\mathbf{x}}-\tilde{f}_{i} \tilde{\xi}_{i}^{0}\right\|^{2}
$$

where $\tilde{f}_{i} \equiv f_{i}\left\|\tilde{\xi}_{i}\right\|$, and $\tilde{\xi}_{i}^{0}=\tilde{\xi}_{i} /\left\|\tilde{\xi}_{i}\right\|$ represents the normalized residual direction for the fault vector $\xi_{i}$.

Minimizing $S P E_{i}$ leads to:

$$
\frac{d S P E_{i}}{d \tilde{f}_{i}}=2{\tilde{\xi_{i}^{0}}}^{T}\left(\tilde{\mathbf{x}}-\tilde{f}_{i}{\tilde{\xi_{i}^{0}}}^{0}\right)=0
$$

or

$$
\tilde{f}_{i}={\tilde{\xi_{i}^{0}}}^{T} \tilde{\mathbf{x}}={\tilde{\xi_{i}^{0}}}^{T}\left(\mathbf{I}-\mathbf{p p}^{T}\right) x
$$

Therefore, the fault vector can be reconstructed as:

$$
f_{i}=\frac{\tilde{f}_{i}}{\left\|\tilde{\xi}_{i}\right\|}=\frac{{\tilde{\xi_{i}^{0}}}^{T}\left(\mathbf{I}-\mathbf{p p}^{T}\right) x}{\left\|\tilde{\xi}_{i}\right\|}
$$

Equation (7) calculates the magnitude of the residual vector. If no fault occurs, then the reconstructed fault will be zero. On the other hand, any significant disturbance or fault will produce a large reconstructed fault value. Thus, this fault construction value can be used to monitor the fault occurrence over time for each input variable, and an islanding event will produce a large reconstructed error according to Equation (7). This will be illustrated in the experimental section.

\section{THE PROPOSED RPCA ALGORITHM}

\section{A. Approximate Linear Dependence Condition}

The approximate linear dependence condition (ALDC) [20] technique is used to update the model with new data samples which can not be represented by a linear combination of previously admitted samples. The ALDC is measured by the approximation error:

$$
\delta_{n+1}=\min \left\|\sum_{i=1}^{n} \alpha_{i} \mathbf{x}_{i}-\mathbf{x}_{n+1}\right\|^{2}
$$

where $\delta_{n+1}$ is the approximate error of the new samples, $\alpha$ is the coefficient. A threshold $\nu$ is needed here to choose the samples, if $\delta_{n+1}$ is less than $\nu$, the new samples can be considered as dependent on the old samples. Further, the ALDC could be computed as:

$$
\delta_{n+1}=\min _{\alpha}\left\{\alpha_{n+1}^{T} \mathbf{K}_{n} \alpha_{n+1}-2 \alpha_{n+1}^{T} \mathbf{n}_{n}+k_{n+1}\right\}
$$

where $\mathbf{K}_{n}=\mathbf{X}_{n} \cdot \mathbf{X}_{n}^{T}, \mathbf{k}_{n}=\mathbf{X}_{n} \cdot \mathbf{x}_{n+1}^{T}, k_{n+1}=\mathbf{x}_{n+1} \cdot \mathbf{x}_{n+1}^{T}$. In order to minimize $\delta_{n+1}$, differentiating it with respect to $\alpha_{n+1}$ yields the linear system solution,

$$
\alpha_{n+1}=\mathbf{K}_{n}^{-1} \mathbf{k}_{n}
$$

Substituting Equation (10) into (9), a recursive ALDC is obtained:

$$
\delta_{n+1}=k_{n+1}-\mathbf{k}_{n}^{T} \alpha_{n+1}=k_{n+1}-\mathbf{k}_{n}^{T} \mathbf{K}_{n}^{-1} \mathbf{k}_{n}
$$

This above approximate error of the new samples in a recursive formula can be applied for new sample selection for on-line updating of the PCA model, thus enables real-time monitoring and fault detection of the grid.

\section{B. Recursive Update of Correlation Matrix}

A significant issue in real-time islanding event detection is to update the PCA model as new data samples are constantly accumulated. A key step of this is to recursively update the correlation matrix when building the PCA model. Let $\mathbf{X}_{l}^{0} \in$ $\mathbb{R}^{N_{l} \times m}$ be the first $l$ data blocks received for building an initial PCA model, and $N_{l}=\sum_{i=1}^{l} n_{i}$ where $n_{i}, i=1, \cdots, l$ is the number of samples in the $i$ th data block, then the mean of each column is given by:

$$
\mathbf{u}_{l}=\frac{1}{N_{l}} \mathbf{1}_{N_{l}} \mathbf{X}_{l}^{0}
$$

where $\mathbf{1}_{N_{l}}=[1, \cdots, 1] \in \mathbb{R}^{1 \times N_{l}}$. Then scale the data to zero mean and unit variance:

$$
\mathbf{X}_{l}=\left(\mathbf{X}_{l}^{0}-\mathbf{1}_{N_{l}}^{T} \mathbf{u}_{l}\right) \Sigma_{l}^{-1}
$$


where $\Sigma_{l}=\operatorname{diag}\left(\sigma_{11}, \cdots, \sigma_{1 m}\right)$ whose $i$ th element of $\sigma_{i m}$ is the standard deviation of the $i$ th data. The correlation matrix is

$$
\mathbf{R}_{l}=\frac{1}{N_{l}-1} \mathbf{X}_{l} \mathbf{X}_{l}^{T}
$$

Suppose $\mathbf{u}_{l}, \mathbf{X}_{l}$ and $\mathbf{R}_{l}$ have been calculated using the above equations when the $l$ blocks of data are collected, the task is to recursively calculate $\mathbf{u}_{l+1}, \mathbf{X}_{l+1}$, and $\mathbf{R}_{l+1}$ when the next data block $\mathbf{X}_{n_{l+1}}^{0} \in \mathbb{R}^{n_{l+1} \times m}$ is made available. The whole data samples collected so far can be expressed as:

$$
\mathbf{X}_{l+1}^{0}=\left[\begin{array}{c}
\mathbf{X}_{l}^{0} \\
\mathbf{X}_{n_{l+1}}^{0}
\end{array}\right]
$$

$\mathbf{u}_{l+1}$ is the mean value of the total $N_{l}+n_{l+1}$ samples, which is related to $\mathbf{u}_{l}$ by the following relation:

$$
\left(\sum_{i=1}^{l+1} n_{i}\right) \mathbf{u}_{l+1}=\left(\sum_{i=1}^{l} n_{i}\right) \mathbf{u}_{l}+\mathbf{1}_{n_{l+1}} \mathbf{X}_{n_{l+1}}^{0}
$$

(16) yields the following recursive calculation:

$$
\mathbf{u}_{l+1}=\frac{N_{l}}{N_{l+1}} \mathbf{u}_{l}+\frac{1}{N_{l+1}} \mathbf{1}_{n_{l+1}} \mathbf{X}_{n_{l+1}}^{0}
$$

Using (15) and (17), the recursive update of $\mathbf{X}_{l+1}$ is given by

$$
\mathbf{X}_{l+1}=\left[\begin{array}{c}
\mathbf{X}_{l} \Sigma_{l} \Sigma_{l+1}^{-1}-\mathbf{1}_{l}^{T} \Delta \mathbf{u}_{l+1} \Sigma_{l+1}^{-1} \\
\mathbf{X}_{n_{l+1}}
\end{array}\right]
$$

Substituting (18) into the correlation matrix $\mathbf{R}_{l+1}$ yields [21]:

$$
\begin{gathered}
\mathbf{R}_{l+1}=\frac{N_{l}-1}{N_{l+1}-1} \Sigma_{l+1}^{-1} \Sigma_{l} \mathbf{R}_{l} \Sigma_{l} \Sigma_{l+1}^{-1}+\frac{N_{l}}{N_{l+1}-1} \Sigma_{l+1}^{-1} \\
\Delta \mathbf{u}_{l+1} \Delta \mathbf{u}_{l+1}^{T} \Sigma_{l+1}^{-1}+\frac{1}{N_{l+1}-1} \mathbf{X}_{n_{l+1}}^{T} \mathbf{X}_{n_{l+1}}
\end{gathered}
$$

Hence, the correlation matrix $\mathbf{R}_{l+1}$ can be updated recursively.

\section{Recursive Process Monitoring}

With the above elaborated techniques for calculating ALDC errors and recursively updating the correlation matrix, a complete flowchart for the proposed power system monitoring approach can be proposed as follows which is illustrated in Fig. 3.

\section{Off-line training}

Step 1: Obtain initial data samples and scale the data.

Step 2: Build the PCA model by computing the covariance matrix and applying PCA to obtain the confidence limit $T_{\alpha}^{2}$ and $Q_{\alpha}$.

\section{On-line updating and monitoring}

Step 3: Collect new samples and scale them.

Step 4 : Compute $T^{2}$ and $Q$ statistics. If $Q>Q_{\alpha}$ or $T^{2}>$ $T_{\alpha}^{2}$, proceed, otherwise, go to step 6 .

Step 5: Fault reconstruction to identify the islanding site and trigger the fault alarm, process end.

Step 6: Update the training data by calculating the ALDC errors to select samples.

Step 7 : Update the PCA model by calculating $R_{l+1}, k, Q_{\alpha}$, $T_{\alpha}^{2}$, set $l=l+1$ and go to step 3 .

The proposed method is a data-driven statistic approach, and the proposed procedure is applicable to different power

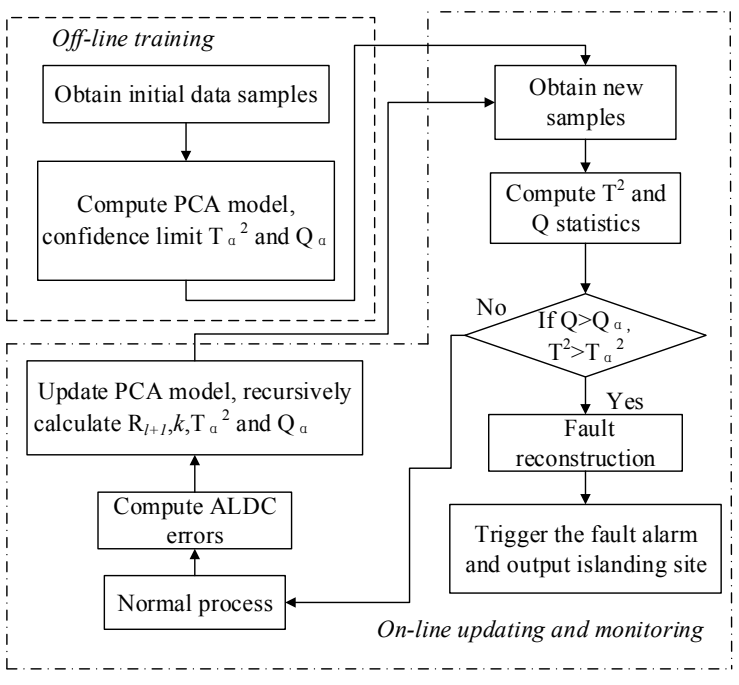

Fig. 3. Flowchart of the systematic RPCA Monitoring Algorithm.

systems. When applied to a new system, data collected from the normal process will first be used as the training data, based on which the confidence limits $T_{\alpha}^{2}$ and $Q_{\alpha}$ will be obtained. Once this step is completed, the trained PCA model can be applied for islanding detection. Unlike conventional methods, the confidence limits are automatically determined using global information from multiple sites, thus the performance can be guaranteed. To alleviate the traffic congestion by transmitting all collected measurements to a central monitoring unit, ALDC algorithm could be implemented at local processing units (e.g. embedded in PMUs), allowing only the data with approximation error exceeding the threshold to be sent to the central unit for updating the PCA model. The on-line PCA model updating part can be implemented at the central unit to receive and process key data samples to update the global model and to perform on-line wide-area system monitoring and fault detection.

\section{EXPERIMENTAL RESULTS AND DISCUSSIONS}

\section{A. Event Analysis}

On 28 September 2012, an inter-connector trip event occurred in the early morning between Great Britain (GB) and France, leading to a $1 \mathrm{GW}$ infeed loss in GB power system. As a result, the main system frequency dropped from $50.08 \mathrm{~Hz}$ to $49.70 \mathrm{~Hz}$, which mistakenly trigged an islanding operation at PMU-2 site (North GB). In the similar case, another trip event occurred later in that day, which was also followed by an islanding event. The detailed frequency plots are given in Fig. 4. As shown, the upper two figures reveal the islanding events, which lasted for 5 hours in the morning, and 1 hour and 23 minutes in the evening. The lower figures are the close-up of the two inter-connector trip events. The bottom left subfigure shows the frequency trip occurred at 02:48:37 at PMU1 site, and the main frequency (red and blue line) reached its minimum of $49.70 \mathrm{~Hz}$ in 10 seconds. The enlarged view in bottom right clearly shows that a small frequency dip first occurred in PMU-3 site at 18:17:15, followed by an islanding 

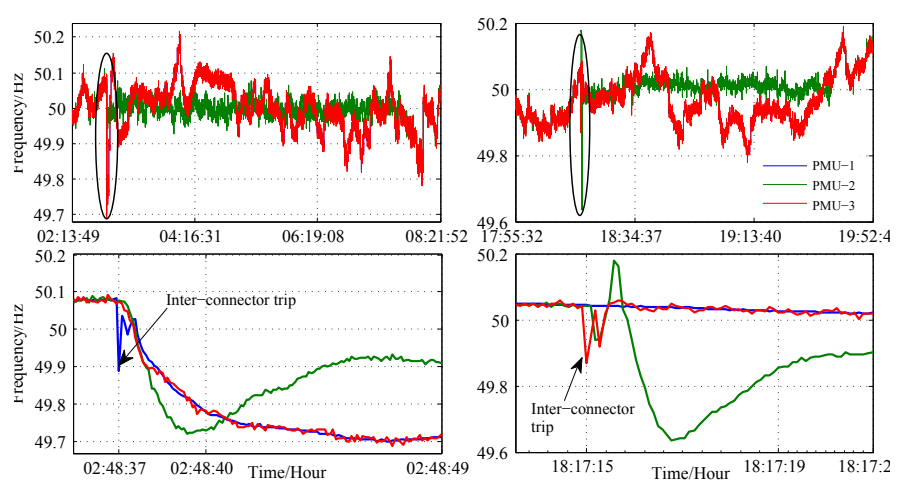

Fig. 4. Frequency plots with two sections of transients and islanding events (upper two sub-figures) and the enlarged view enclosed by the ellipses (lower two sub-figures) on 28th Sep. 2012

operation at PMU-2 site, while the main frequency was kept in normal range.

\section{B. Comparisons of ROCOF and PCA results}

The data of the two sections have been processed using ROCOF with different windows of 5 cycles ( 0.1 second) and 50 cycles ( 1 second), the results are shown in Fig. 5 and Fig. 6 . The black dashed line presents the pre-determined threshold of $0.125 \mathrm{~Hz} / \mathrm{s}$ for the northern GB power grid [22]. Both of
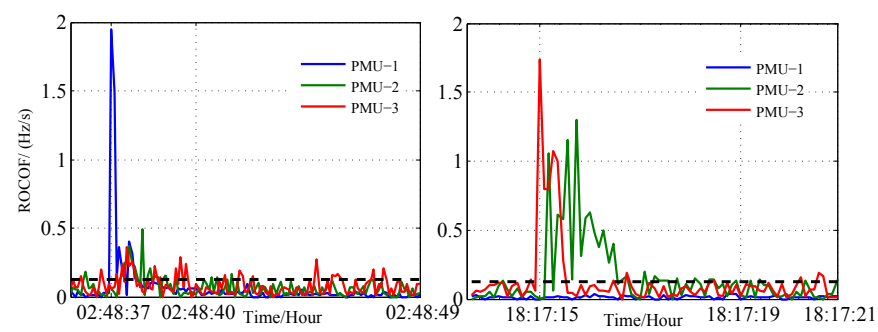

Fig. 5. ROCOF results of two sections based on 5 cycles.

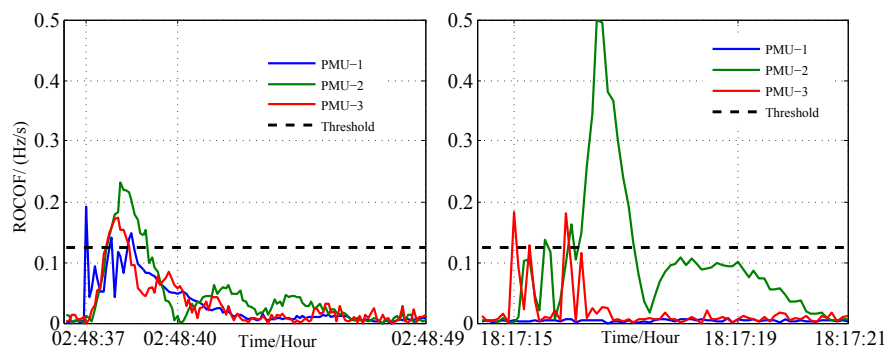

Fig. 6. ROCOF results of two sections based on 50 cycles.

the two figures show that ROCOF result of PMU-1 exceeded the threshold for the first section, indicating the frequency dip event occurred. However, calculation based on small window ( 5 cycles) leads to larger ROCOF values and it is very sensitive to noise. As seen in Fig.5, many points exceeded the threshold, causing false alarms. The violation at time instant 02:48:37 in the first transient comes from PMU-1, and the violations of all the PMUs only lasted for several seconds. In fact, the islanding situation which lasted for hours can not be verified from the result.

For the result based on 50 cycles shown in Fig.6, false violations has been reduced due to a bigger ROCOF window. The same time instant of the event can be detected based on the PMU-1 data. However, all PMUs in the first section and PMU-2 and PMU-3 in the second section have revealed a period of violation of the threshold, which show that the islanding site can not be accurately identified directly from the ROCOF results. This analysis reveals that the ROCOF method can detect violations but failed to distinguish the islanding event from the frequency transient, thus was unable to identify the islanding site. In summary, the disadvantages of traditional ROCOF method include: 1) calculation window and sampling rate of the PMU data affect the ROCOF results, 2) threshold needs to be determined according to the strength of the real system, 3) can not distinguish between frequency transients and islanding events, 4) impossible to identify the islanding site and 5) fail to detect the duration and return-to-main time of the islanding event.

Data consisting of 6 PMUs are used for conventional PCA monitoring. The results are given in Fig. 7, with two statistics $T^{2}$ and $Q$ and the $99 \%$ confidence limits. Fig. 7 shows the suc-
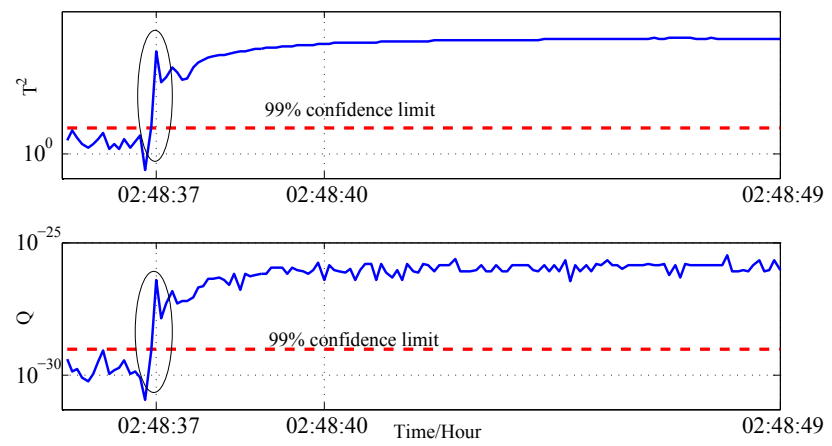

Fig. 7. Successful PCA monitoring result under $99 \%$ confidence limit.

cessful and accurate detection of frequency dip event (enclosed by the ellipse), and the exceeding values after the inception point show continuous change in the data variance which implies the unsynchronization. Further islanding identification can be carried out by fault reconstruction method. Compare to traditional ROCOF method, PCA can process the multiple PMU data as a whole data matrix and respond very quickly (detailed detection time will be compared with RPCA later), without considering the strength of the actual power system. Moreover, the monitoring charts using $T^{2}$ and $Q$ statistics do not need to consider the calculation cycles and sampling rate.

In order to demonstrate the problem of standard PCA method in detecting faults for time varying processes, another group of normal data recorded for a much longer period of time (50 minutes) before the transient occurred were used to build the PCA model, then the model was applied to the same testing data. The test results are shown in Fig. 8. The results reveal a different behaviour of the static PCA model on the same testing data but trained with different normal data samples. Although the first peak values (enclosed in the ellipses) occurred at the same time instant, no correct response 

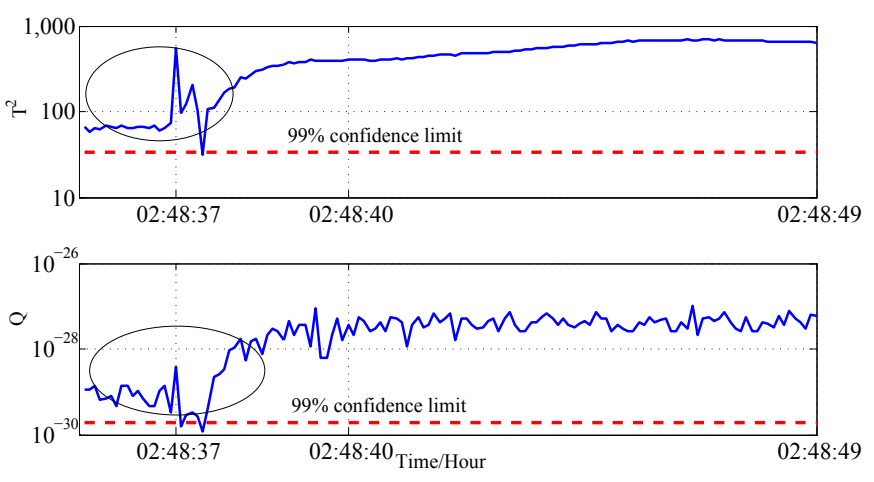

Fig. 8. Failed PCA monitoring result under $99 \%$ confidence limit.

TABLE I

COMPARISON STATISTICS OF DIFFERENT THRESHOLDS

\begin{tabular}{|c|c|c|c|c|c|c|}
\hline Threshold & \multicolumn{7}{|c|}{ Number of samples for updating (n) } & RMSE \\
\hline $0.1 *{ }_{m}$ & 18 & 37 & 40 & 80 & 70 & 0.0835 \\
\hline $\mathbf{0 . 3}^{*} \delta_{m}$ & $\mathbf{5}$ & $\mathbf{4}$ & $\mathbf{2 7}$ & $\mathbf{5 2}$ & $\mathbf{3 4}$ & $\mathbf{0 . 1 4 3 0}$ \\
\hline $0.5^{*} \delta_{m}$ & 3 & 9 & 1 & 24 & 8 & 0.2411 \\
\hline $0.7^{*} \delta_{m}$ & 2 & 1 & 16 & 1 & 1 & 0.4935 \\
\hline $0.9^{*} \delta_{m}$ & 2 & 1 & 16 & 1 & 1 & 0.4935 \\
\hline Threshold & \multicolumn{7}{|c|}{ Elapsed time calculating each iteration (second) } \\
\hline $0.1^{*} \delta_{m}$ & 0.1474 & 0.2175 & 0.2614 & 0.4793 & 0.5519 \\
\hline $\mathbf{0 . 3} \delta_{m}$ & $\mathbf{0 . 1 4 7 5}$ & $\mathbf{0 . 1 5 5 9}$ & $\mathbf{0 . 1 5 9 3}$ & $\mathbf{0 . 1 9 0 1}$ & $\mathbf{0 . 1 9 5 2}$ \\
\hline $0.5^{*} \delta_{m}$ & 0.1507 & 0.1492 & 0.1806 & 0.1779 & 0.2021 \\
\hline $0.7^{*} \delta_{m}$ & 0.1545 & 0.1551 & 0.1457 & 0.1707 & 0.1731 \\
\hline $0.9^{*} \delta_{m}$ & 0.1509 & 0.1537 & 0.1540 & 0.1553 & 0.1545 \\
\hline
\end{tabular}

was generated for other samples and all values are above the limits even before the transient inception time (02:48:37). This reflects the defect of static PCA model that due to the time-varying characteristic of power systems, process variables often experience slow but normal changes, the confidence limits calculated based on old training data will no longer be suitable for new data samples. Therefore, it is difficult to monitor a time-varying system based on a static model. This is also the motivation of this paper in proposing a recursive PCA method to keep the PCA model updated in real time.

\section{Sample Reduction by ALDC}

The first step of the recursive method is to use ALDC algorithm to check the independence of the new data. The first 600 samples of the PMU data were divided into six parts, each of the remaining parts went through an independence check against the first group. After updating, the size of the training data is reduced to 187 samples instead of 600 samples, thus the computational burden is reduced. The selection of the threshold used in the independence check is a tradeoff between the estimation accuracy and computing time. Comparisons of the 'Number of samples for updating (n)', 'Root mean square error (RMSE)' and the 'Elapsed time (s)' for calculating each block of data under different thresholds are shown in Table.1. $\delta_{m}$ represents the maximum value of the approximation error. High accuracy is expected (with RMSE of 0.0835 ) when the threshold was chosen to be small enough (10\% of the maximum value) as more data were added to the training sample pool. The statistics are almost the same for the thresholds of $0.7^{*} \delta_{m}$ and $0.9^{*} \delta_{m}$. This is because the data numbers with relatively higher approximation error is very limited, thus these large thresholds do not have any significant impact on the choice of training data. Given this consideration and also the trade-off of the three aspects listed in Table I, $0.3 * \delta_{m}$ is chosen as the threshold for acceptable computational time and small RMSE. It should be noted that the threshold is obtained by calculating the linear relationship between the newly arrived samples and existing training samples under normal operation conditions. This threshold once chosen is usually fixed and robust for the same bulk power system. The following experimental section shows that the proposed RPCA based on-line monitoring scheme with this above chosen ALDC threshold is capable of detecting the violations accurately appearing in abnormal PMU data and the islanding event, regardless of load and DG operating conditions.

\section{RPCA Modelling Results}

The initial result without model updating is given in Fig. 9 , the $T^{2}$ and $Q$ statistics demonstrate an obvious trend of violating the control limits between 500th to 600th samples. The samples after 600th all exceeded the control limits in both charts, as shown in the circled area. On contrast, the results produced from the model updating is given in Fig.10, which shows that it effectively captured this trend of slow changing in operation conditions in the model and the number of false alarms is significantly reduced. Confidence level can even be switched to $99.5 \%$ if higher accuracy is needed. Finally,

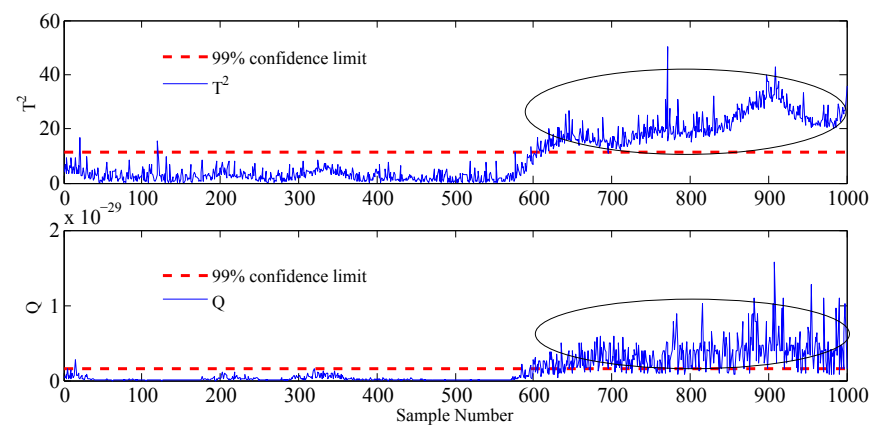

Fig. 9. The monitoring result of PCA for normal PMU data.

the proposed RPCA was verified by monitoring the transients. With model updating scheme, the detection results of the inter-connector failure are shown in Fig.11. The calculated $T^{2}$ and $Q$ statistics have successfully detected the occurring time of the inter-connector failure (02:48:37), as enclosed in the solid ellipse. In addition, an instant of return-to-main situation has been detected during islanding event by the $Q$ statistic after 02:48:40 (enclosed in the dashed ellipse), which was however not detected using conventional PCA. Furthermore, Fig.12 reveals the successful detection of return-to-main time (07:43:57) when the islanding event ended and PMU-2 site returned to the main and was in synchronization with the utility grid. This situation was however not detected when the ROCOF method was used. Once an islanding event was detected, 


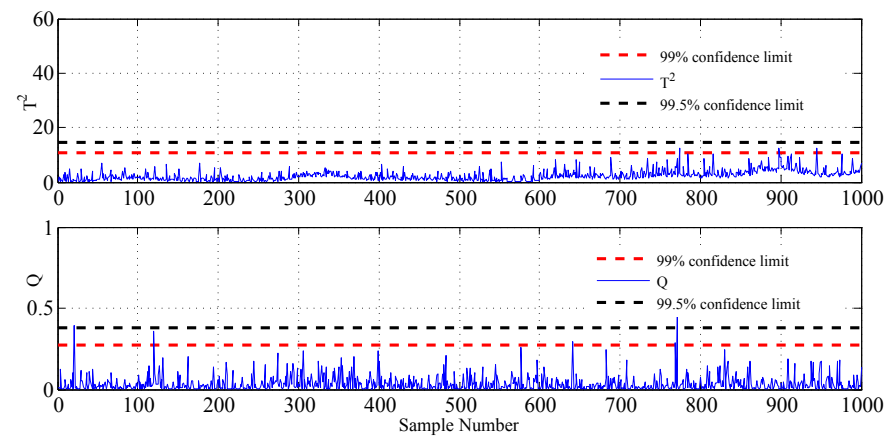

Fig. 10. The normal data monitoring result of RPCA.

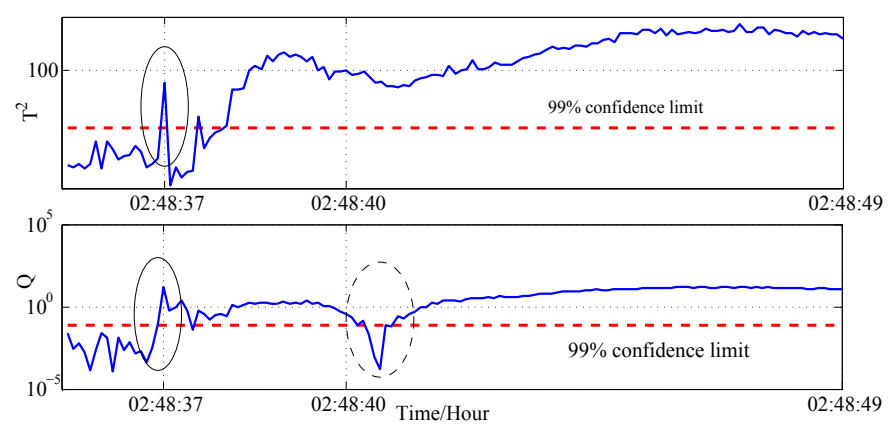

Fig. 11. The transients monitoring result of RPCA.

another important issue was to identify the islanding site. As shown in Fig.13, using the fault reconstruction method, PMU5 is shown to have a dramatically large fault reconstruction error which indicates that this variable contributes the most in the fault reconstruction, and the corresponding site is therefore the islanding site.

\section{E. Discussion}

The proposed RPCA method for islanding detection involves off-line PCA modelling, on-line updating and monitoring procedure. The implementation of the proposed method requires the consideration of the response time, false alarm rate (FAR) and fault detection ability (FDA). The comparisons of traditional ROCOF, PCA and RPCA are given in Table II.

In summary, traditional ROCOF method is very easy to implement and can quickly respond to any violating point. However, it is not reliable for complex situations and sensitive to noise and measurement errors. Moreover, ROCOF fails to identify islanding site and detect the return-to-main time. On the other hand, PCA methods can process the multivariate data in a whole matrix thus the FDA could be significantly increased while FAR is decreased for short term monitoring. RPCA is designed for time-varying systems by which the timevarying trend can be captured in time. In addition, ALDC is used to reduce the training data, this allows the computation time to be further reduced within 2 seconds to meet the IEEE standard. In terms of FDA and FAR, RPCA generally outperform PCA and the traditional ROCOF as demonstrated in the experimental results. In particular, RPCA can capture the time-varying characteristic of the normal process, thus high

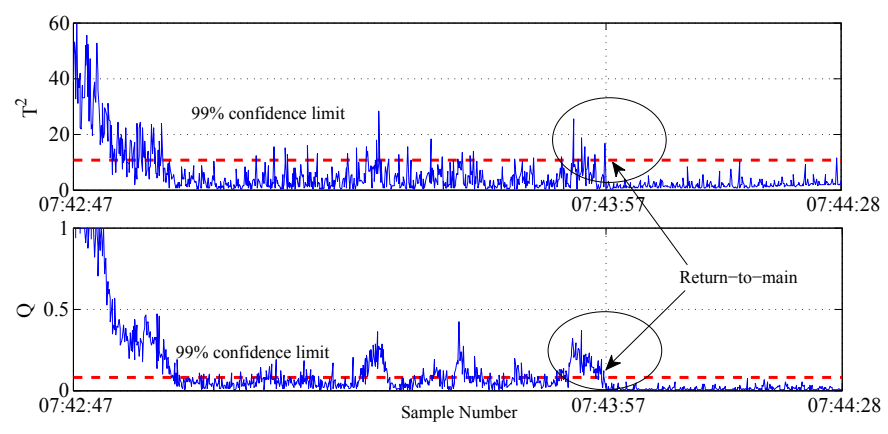

Fig. 12. The monitoring result of RPCA for return-to-main detection.

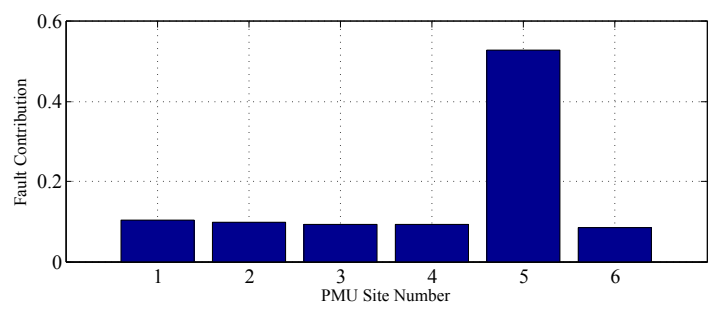

Fig. 13. Fault reconstruction result for islanding site identification.

accuracy detection results for islanding situation can provide the operator with more reliable information from the prospect of wide-area power system monitoring framework. Other nonislanding events such as load switching, load shedding and inter-connector trip often cause sudden frequency changes and deviations. This will lead to violations of the statistics limits in the PCA and RPCA methods, but such violations normally will not last for long if no islanding event occurs. This has been clearly demonstrated in the above experimental section.

\section{CONCLUSION}

PCA based methods including both static and recursive approach have been proposed in this paper to adaptively monitor transient situations in power systems with significant penetration of renewable energies and a number of distribution networks, while the system do not have sufficient inertia to limit frequency divergences in the event of system fault/stress. Comparisons of ROCOF with PCA and RPCA monitoring results have demonstrated the efficacy of the proposed methods. Traditional methods such as ROCOF is not reliable for timevarying process and is sensitive to noise. More importantly, the identification of islanding site is not possible for ROCOF method due to the lack of analysis between process variables. PCA based methods have thus been applied to wide-area monitoring system at the distribution level. Accurate event occurring time could be pinpointed by $T^{2}$ and $Q$ statistics, and the islanding site could be identified through the fault reconstruction method. The advantages of the proposed PCA methods in comparison with traditional islanding approaches include: 1) effective and fast on-line monitoring, 2) avoid the situation that reference site itself is non-synchronous, and 3) extend the functionality of PMU devices and provide a useful 
TABLE II

COMPARISONS OF ROCOF, PCA AND RPCA

\begin{tabular}{|c|c|c|c|c|c|}
\hline & \multicolumn{2}{|c|}{ ROCOF } & \multicolumn{2}{|c|}{ PCA } & RPCA \\
\hline \multirow{2}{*}{$\begin{array}{c}\text { Response time } \\
\text { /seconds (off-line) }\end{array}$} & 3 PMUs & 0.004686 & \multicolumn{2}{|c|}{0.1866} & 0.4573 \\
\hline & 6 PMUs & 0.004686 & \multicolumn{2}{|c|}{0.1880} & 0.4903 \\
\hline \multirow{2}{*}{$\begin{array}{l}\text { Response time } \\
\text { /seconds (on-line) }\end{array}$} & 3 PMUs & 0.000022 & \multicolumn{2}{|c|}{0.0038} & 0.0256 \\
\hline & 6 PMUs & 0.000023 & \multicolumn{2}{|c|}{0.0044} & 0.0312 \\
\hline \multirow{2}{*}{ False alarm rate } & 5 cycles & $5.6 \%$ & Short-term & $0 \%$ & \multirow{2}{*}{$0.1 \%$} \\
\hline & 50 cycles & $1.7 \%$ & Long-term & $12.4 \%$ & \\
\hline \multirow{2}{*}{ Fault detection ability } & 5 cycles & $12.97 \%$ & Short-term & $100 \%$ & \multirow{2}{*}{$100 \%$} \\
\hline & 50 cycles & $14.62 \%$ & Long-term & $100 \%$ & \\
\hline Advantages & \multicolumn{2}{|c|}{$\begin{array}{l}\text { 1. Fast response. } \\
\text { 2. Easy to implement. }\end{array}$} & \multicolumn{2}{|c|}{$\begin{array}{l}\text { 1. Fast online response. } \\
\text { 2. Theoretically calculated threshold. } \\
\text { 3. Intuitive control charts. }\end{array}$} & $\begin{array}{l}\text { 1. High accuracy for both short } \\
\text { and long term monitoring. } \\
\text { 2. Simpler presentation with } T^{2} \text {. }\end{array}$ \\
\hline Disadvantages & \multicolumn{2}{|c|}{$\begin{array}{l}\text { 1. Not reliable for time-varying system. } \\
\text { 2. Difficult to determine the empirical threshold. } \\
\text { 3. Impossible to identify islanding site. }\end{array}$} & \multicolumn{2}{|c|}{$\begin{array}{l}\text { 1. Not suitable for time-varying } \\
\text { system and long-term monitoring. } \\
\text { 2. High false alarm rate. }\end{array}$} & $\begin{array}{l}\text { 1. Complex off-training procedure. } \\
\text { 2. Slower response than } \\
\text { traditional method. }\end{array}$ \\
\hline
\end{tabular}

method for more intelligent information system in the future grid.

To handle the time-varying situations, the PCA method has been extended to RPCA for islanding detection. The experimental results with and without updating training data have shown the capability of RPCA in capturing the timevarying characteristic of the power system. In order to reduce the computation time, ALDC is used, thus only data containing useful and different information is added to the training data. Although, RPCA based monitoring has a slower response than ROCOF and PCA, the merits of excellent fault detection ability and nearly zero false alarm rate will help engineers to identify the faults in time, and a common wide-area view of the power system and early warning of the network abnormality can also be provided. Therefore, the risk of damaging utility plants and customer connected equipments due to unintentional islanding events can be greatly reduced.

\section{ACKNOWLEDGEMENT}

The authors wish to acknowledge Scottish \& Southern Energy Power Distribution Ltd. for the assistance they have rendered in support of this work. Special thanks to $\mathrm{X}$. $\mathrm{Xu}$ from Tianjing University for the valuable discussions during his visit to Queen's University Belfast. In addition, the authors would like to thank the handling editor and the reviewers for their constructive comments.

\section{REFERENCES}

[1] D. Laverty, D. Morrow, R. Best, and M. Cregan, "Anti-islanding detection using synchrophasors and internet protocol telecommunications," in Innovative Smart Grid Technologies (ISGT Europe), 2011 2nd IEEE PES International Conference and Exhibition on. IEEE, 2011, pp. 1-5.

[2] "Wind energy in ireland statistics," http://www.iwea.com/index.cfm/ page/windenergyfacts, 2013, accessed: 27th Feb 2014.

[3] P. Crossley, F. Ilar, and D. Karlsson, "System protection schemes in power networks: existing installations and ideas for future development," in Developments in Power System Protection, 2001, Seventh International Conference on (IEE). IET, 2001, pp. 450-453.

[4] W. Gao et al., "Comparison and review of islanding detection techniques for distributed energy resources," in 2008 40th North American power symposium, 2008, pp. 1-8.

[5] D. M. Laverty, R. J. Best, and D. J. Morrow, "Loss-of-mains protection system by application of phasor measurement unit technology with experimentally assessed threshold settings," IET Generation, Transmission \& Distribution, pp. 146-153, 29 Jan 2015.
[6] D. M. Laverty, R. J. Best, P. Brogan, I. Al Khatib, L. Vanfretti, and D. J. Morrow, "The openpmu platform for open-source phasor measurements," IEEE Transactions on Instrumentation and Measurement, vol. 62, no. 4, pp. 701-709, 2013.

[7] Z. Lin, T. Xia, Y. Ye, Y. Zhang, L. Chen, Y. Liu, K. Tomsovic, and T. Bilke, "Power system islanding detection based on wide area measurement systems," in Intelligent System Application to Power Systems (ISAP), 2011 16th International Conference on. IEEE, 2011, pp. 1-6.

[8] X. Wang, W. Freitas, and W. Xu, "Dynamic non-detection zones of positive feedback anti-islanding methods for inverter-based distributed generators," Power Delivery, IEEE Transactions on, vol. 26, no. 2, pp. $1145-1155,2011$.

[9] A. Khamis, H. Shareef, E. Bizkevelci, and T. Khatib, "A review of islanding detection techniques for renewable distributed generation systems," Renewable and Sustainable Energy Reviews, vol. 28, pp. 483493, 2013.

[10] Y. Guo, K. Li, and D. Laverty, "A statistical process control approach for automatic anti-islanding detection using synchrophasors," in Power and Energy Society General Meeting, 2013 IEEE. IEEE, 2013, pp. $1-6$.

[11] X. Liu, L. David, K. Li, M. John, and M. Sean, "Principal component analysis of wide area phasor measurements for islanding detection a geometric view," IEEE Transactions on Power Delivery, vol. PP, no. 99, pp. 1-10, 2015.

[12] Y. Guo, K. Li, and D. Laverty, "Loss-of-main monitoring and detection for distributed generations using dynamic principal component analysis," Journal of Power and Energy Engineering, vol. 2, no. 04, p. 423, 2014.

[13] X. A. Liu, D. Laverty, and R. Best, "Islanding detection based on probabilistic pca with missing values in pmu data," in PES General Meeting-Conference \& Exposition, 2014 IEEE. IEEE, 2014, pp. $1-6$.

[14] E. N. Association, Engineering Recommendation G59/3: Recommendations for the Connection of Generating Plant to the Distribution Systems of Licensed Distribution Network Operators. London, U.K., 1st Sept. 2013.

[15] I. Jolliffe and MyiLibrary, Principal component analysis. Wiley Online Library, 2002, vol. 2.

[16] I. Dryden and K. Mardia, Statistical shape analysis. John Wiley \& Sons New York, 1998, vol. 4.

[17] J. Kresta, J. Macgregor, and T. Marlin, "Multivariate statistical monitoring of process operating performance," The Canadian Journal of Chemical Engineering, vol. 69, no. 1, pp. 35-47, 1991.

[18] N. Tracy, J. Young, and R. Mason, "Multivariate control charts for individual observations," Journal of Quality Technology, vol. 24, no. 2, 1992.

[19] J. E. Jackson and G. S. Mudholkar, "Control procedures for residuals associated with principal component analysis," Technometrics, vol. 21, no. 3, pp. 341-349, 1979.

[20] J. Tang, W. Yu, T. Chai, and L. Zhao, "On-line principal component analysis with application to process modeling," Neurocomputing, vol. 82, pp. 167-178, 2012.

[21] W. Li, H. H. Yue, S. Valle-Cervantes, and S. J. Qin, "Recursive pca for adaptive process monitoring," Journal of process control, vol. 10, no. 5, pp. 471-486, 2000. 
[22] X. Ding and P. A. Crossley, "Islanding detection for distributed generation," in Power Tech, 2005 IEEE Russia. IEEE, 2005, pp. 1-4.

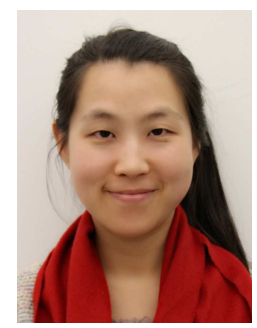

Y. Guo (S'13) received the B.Sc. degree in Information Engineering, the M.Sc degree in Optoelectronic engineering from Chongqing University, Chongqing, China, in 2008 and 2011, respectively. She is currently pursuing the Ph.D. degree with the School of Electronics, Electrical Engineering and Computer Science, Queens University Belfast, U.K.

Her current research interests include statistical process control, islanding detection and power system wide-area monitoring.

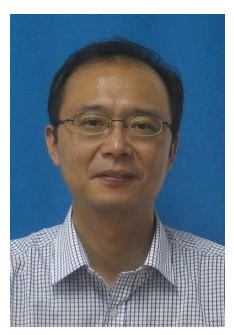

K. Li (M'05-SM'11) received the B.Sc. degree in Industrial Automation from Xiangtan University, Hunan, China, in 1989, the M.Sc. degree in Control Theory and Applications from Harbin Institute of Technology, Harbin, China, in 1992, and the Ph.D. degree in Control Theory and Applications from Shanghai Jiaotong University, Shanghai, China, in 1995.

Between 1995 and 2012, he worked at Shanghai Jiaotong University, Delft University of Technology and Queens University Belfast as a research fellow. Since 2002, he was a Lecturer, a Senior Lecturer (2007) and a Reader (2009) with the School of Electronics, Electrical Engineering and Computer Science, Queens University Belfast, Belfast, U.K, and he becomes a Professor from 2011. His research interests include nonlinear system modeling, identification, and control, and bio-inspired computational intelligence, with applications to energy and power systems, smart grid, electric vehicles, and polymer processing. He is particularly interested in the development of advanced control technologies for decarbonizing the whole energy systems from head to tail, including a new generation of low cost minimal-invasive monitoring system and intelligent control platform for energy intensive industries. He is the author or co-author of more than 200 articles, and edited or co-edited over 10 conference proceedings.

Dr Li chairs the IEEE UKRI Control and Communication Ireland chapter, and was the Secretary of the IEEE UK \& Ireland Section. He is a visiting professor of Harbin Institute of Technology, Shanghai University, and Ningbo Institute of Technology of Zhejiang University. He also held visiting fellowship or visiting professorship at National University of Singapore, University of Iowa, New Jersey Institute of Technology, Tsinghua University, and Technical University of Bari, Taranto.

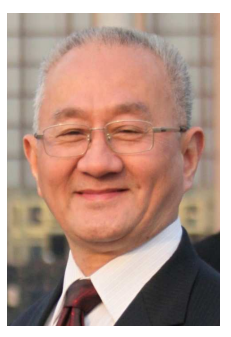

Y. Xue Professor Yusheng XUE received M.Sc. degree in Electrical Engineering from EPRI, China in 1981 and PhD degree from the University of Liege, Belgium in 1987. He was elected an academician of Chinese Academy of Engineering in 1995. He is now the Honorary President of State Grid Electric Power Research Institute (SGEPRI or NARI), China. He holds the positions of Adjunct Professor in many universities in China and a conjoint professor of the University of Newcastle in Australia. He was also an honorary professor of the University of Queensland, Australia. He is the Editor-in-Chief of the Journal of Automation of Electric Power System (in Chinese) and that of the Journal of Modern Power System and Clean energy (in English), and a member of Editorial Board of IET Generation, Transmission \& Distribution, and Chairman of Technical Committee of Chinese National Committee of CIGRE since 2005.

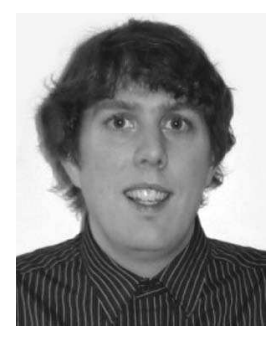

D. M. Laverty (M'10) was born in Belfast, Northern Ireland, in 1984. He received the M.Eng and Ph.D. degrees from Queens University Belfast, Belfast, UK, in 2006 and 2010 respectively. His placements as an undergraduate were with Northern Ireland Electricity and Universitt Paderborn, Germany.

Since 2011 he has been a Lecturer with the Electrical Power and Energy Research Cluster at Queens University Belfast, Belfast, UK. His current research interests are in power system measurements, anti-islanding detection, phasor measurements, and Smart Grid telecommunications, messaging and security.

Dr. Laverty is a member of the IEEE and volunteers with the Institution of Engineering and Technology (IET). 